\title{
Centrocestus formosanus (Opisthorchiida: Heterophyidae) como causa de muerte de alevines de tilapia gris Oreochromis niloticus (Perciforme: Cichlidae) en el Pacífico seco de Costa Rica
}

\author{
Donald Arguedas Cortés ${ }^{1}$, Gaby Dolz ${ }^{2}$, Juan J. Romero Zúñiga ${ }^{2}$, Ana E. Jiménez Rocha ${ }^{3}$ \& \\ Dennis León Alán ${ }^{4}$ \\ 1. Laboratorio de Biología, Universidad Técnica Nacional, Guanacaste, Costa Rica. Tel: 26740268-ext 3, Fax: 26740290; \\ darguedas@utn.ac.cr \\ 2. Programa de Investigación en Medicina Poblacional, Escuela de Medicina Veterinaria, Universidad Nacional, \\ Heredia, Costa Rica; gabyd@medvet.una.ac.cr \\ 3. Laboratorio de Parasitología, Escuela de Medicina Veterinaria, Universidad Nacional, Heredia, Costa Rica; \\ anaj@medvet.una.ac.cr \\ 4. Laboratorio de Helmintología, Facultad de Microbiología, Universidad de Costa Rica, San José, Costa Rica; \\ denleoncr@yahoo.com
}

Recibido 27-VII-2009. Corregido 21-VI-2010. Aceptado 23-VII-2010.

\begin{abstract}
Centrocestus formosanus (Opisthorchiida: Heterophyidae) as a cause of death in gray tilapia fry Oreochromis niloticus (Perciforme: Cichlidae) in the dry Pacific of Costa Rica. Centrocestus formosanus is a zoonotic trematode from Asia and has been mainly associated as cause of death of cultured fish. To identify pathogen trematode species in tilapia fry (Oreochromis niloticus) and to determine mollusks hosting these parasites, freshwater mollusks were collected from tilapia cultured ponds and experimental infections were carried out with tilapia fries and different mollusk species. A total of 907 freshwater mollusks were obtained from tilapia ponds and were identified to species level, four gastropods and one bivalve were determined: Melania tuberculata, Melanoides turricula, Pomacea flagellata, Haitia cubensis and Anodontiles luteola. For the first time, the presence of M. turricula and H. cubensis are reported in Costa Rica. Seven morphotypes of cercariae (Xifiodiocercaria, Equinostoma, Oftalmocercaria, Parapleurolofocercus, Cistocerca, Furcocercaria and Leptocercaria) parasitizing all five species of mollusks were found, all of distome type. Experimental exposure of tilapia fry to $M$. tuberculata demonstrated that the parapleurolofocercus morphotype found in the mollusk is in accordance with the finding of $C$. formosanus in tilapia fry. An abundance and mean intensity of 1018-1027 digeneans per gill in each exposed fish was determined. Centrocestus formosanus is reported for the first time in Costa Rica, for which the primary and secondary intermediate hosts were also determined. Rev. Biol. Trop. 58 (4): 1453-1465. Epub 2010 December 01.
\end{abstract}

Key words: Centrocestus formosanus, Oreochromis niloticus, tilapia fry, freshwater mollusk, Costa Rica.

La acuacultura, como actividad industrial, ha experimentado un notable desarrollo global durante los últimos años, con un aumento del $200 \%$ en el periodo 2000-2004 en Costa Rica (INCOPESCA 2005) y representa a nivel mundial un $31 \%$ de la producción pesquera mundial (Hepher \& Pruginin 2004). Costa Rica no ha sido la excepción, esta actividad incrementó de 9356 toneladas métricas (TM) en el año 2000, a 18 000TM en el 2005, en donde la tilapia es el pez que más se cultiva en el país, en comparación con los demás organismos producidos en cultivos acuáticos (INCOPESCA 2006). En términos generales, se producen anualmente 26000TM de tilapia en el país (FAO 2005), lo que genera, por concepto de ventas, cerca de 27.5 millones de dólares anuales (INCOPESCA 2006), siendo Guanacaste la provincia 
donde se encuentran instaladas la empresas que cultivan el 95\% de la producción total (INCOPESCA 2005).

La tilapia gris (Oreochromis niloticus) puede ser parasitada por distintos organismos en cultivos acuícolas (Conroy \& Conroy 1998). Los parásitos pueden afectar piel, musculo, cavidad branquial y branquias, ojos, hígado, bazo, riñones, vejiga natatoria e intestino, que con altas infecciones podrían llegar a producir emaciación y mortalidades masivas en cultivos (Paperna 1991). Los tremátodos digéneos han sido catalogados de gran importancia económica por los efectos negativos que produce en peces de cultivos dulceacuícolas y silvestres (Knoff et al. 2001). Estos son gusanos planos con un ciclo biológico heteroxeno y que, generalmente, requieren de un molusco (caracol o bivalvo) como primer hospedador intermediario, un pez como segundo hospedero intermediario y un ave piscívora, pez o mamífero como hospedero final (Healy 1985, Soulsby 1987). Los parásitos adultos se localizan en el intestino del hospedador definitivo y rara vez están implicados como causa de enfermedad o muerte (Cremonte \& Martorelli 1998). Las aves, mamíferos o peces parasitados, defecan los huevos embrionados al agua del estanque, liberando un miracidio, que posteriormente ingresa al molusco y se transforma en cercaria con morfotipo característico (Schmidt \& Roberts 2000); una vez ahí, se enquista en el pez y es llamada metacercaria. Estas son potencialmente patógenas para los peces (Noga 2000) y la mortalidad es más frecuente en alevines menores de 30 días, manifestando nadado lento en espiral, ligero arqueamiento del cuerpo y muerte durante su permanencia en estanques de cultivo (Paperna 1996, Pironet \& Jones 2000).

Existen pocos estudios ictiopatológicos en Costa Rica. Matamoros (1982) encontró metacercarias enquistadas en ojos de tilapias cultivadas en Cañas, Guanacaste, reportando que se trataba de Diplostomum sp., no obstante, en la lista de helmintos de Costa Rica (Rodríguez et al. 2004a, b) no se menciona este hallazgo. Muñoz (2001) reporta la muerte de alevines de tilapias ocasionada por tremátodos digéneos, encontrando dos quistes de metacercarias, morfológicamente diferentes, en branquias e hígado, a los que llamó morfotipo 1 y 2; sin embargo, no logró identificar las especies de parásitos y los moluscos que portaban las cercarias.

El presente trabajo tuvo como objetivo identificar las especies de tremátodos digéneos patógenos para alevines de tilapia, así como los moluscos hospedadores de dichos parásitos; además, determinar las especies de moluscos presentes en estanques de cultivo de tilapia y los morfotipos de cercarias presentes en los moluscos.

\section{MATERIALES Y MÉTODOS}

Área de estudio: Para los muestreos de los moluscos, se seleccionaron siete localidades, una en cada provincia de Costa Rica: 1.) Estación Experimental Acuícola Enrique Jiménez Núñez, de INCOPESCA, en la localidad de Cañas, Guanacaste, $\left(10^{\circ} 20^{\prime} 00^{\prime \prime} \mathrm{N}\right.$; 85'05'00" W), 2.) Finca productora "Cuestillas", en San Carlos de Alajuela $\left(10^{\circ} 13^{\prime} 13^{\prime \prime} \mathrm{N} ; 4^{\circ} 35^{\prime} 20^{\prime}\right.$ " $\mathrm{W})$, ambas productoras de semilla de tilapia de la especie $O$. niloticus; 3 .) Estación Experimental Acuícola 28 Millas (1000'29" N; 85¹5'06" W) en la provincia de Limón y fincas acuícolas comerciales extensivas y de subsistencia, ubicadas en las localidades de 4.) Aserrí de San José (0947 ‘48” N; 8402'55” W), 5.) Barva de Heredia (09.59'17" N; 8409'14” W), 6.) Paraíso de Cartago (0948'59" N; 8342'09" W) y 7.) Jacó de Puntarenas (09 $56^{\circ} 55^{\prime \prime} \mathrm{N}$; 84²3'36" W).

Recolecta de moluscos: El muestreo malacológico se realizó entre agosto y diciembre de 2004. En cada localidad se analizó un estanque. Los estanques en las localidades 1 y 2 presentaron un área total de $300 \mathrm{~m}^{2}$, mientras que en las otras localidades de $200 \mathrm{~m}^{2}$. La recolecta se llevó a cabo según Rodríguez (1980) para estudios de bentos. En los estanques grandes se recolectaron moluscos en siete cuadrantes y en los estanques pequeños en cinco cuadrantes 
de $2 \mathrm{~m} \times 2 \mathrm{~m}$, que fueron distribuidos en diversos puntos del estanque, cada $4 \mathrm{~m}$ lineales, formando una "S" imaginaria, desde el extremo en donde se ubicó la entrada del agua, hasta la salida de la misma y manteniendo una relación proporcional entre los cuadrantes que se encontraban en los bordes y los centros. Se consideró como borde toda área comprendida desde la orilla hasta una distancia de $2 \mathrm{~m}$ hacia el centro. Las partes centrales correspondieron al resto del área que se ubicó a una distancia mayor de $2 \mathrm{~m}$ de la orilla.

La recolecta de moluscos se realizó en forma directa (manual) con una malla de cedazo de $1 \mathrm{~mm}$ de luz, tomando una muestra de lodo de cada cuadrante a una profundidad promedio de $85 \mathrm{~cm}$. Los organismos vivos extraídos de cada estanque fueron depositados en recipientes plásticos, debidamente etiquetados y trasladados en una hielera al Laboratorio de Biología del antiguo Colegio Universitario para el Riego y el Desarrollo del Trópico Seco (CURDTS), donde se mantuvieron en recipientes con aireación hasta su cuantificación e identificación.

Identificación de moluscos y cercarias: Los moluscos se clasificaron utilizando estereoscopía y la clave genérica para la identificación de gastrópodos de agua dulce de Burch \& Cruz-Reyes (1987). Para visualizar las cercarias en los moluscos, se utilizó la técnica de análisis de aplastamiento, estereoscopía y microscopio de luz (40X y 100X). Las cercarias fueron clasificadas con base en el número de ventosas (Krull 1986) y para la clasificación de los morfotipos se utilizó la guía de Yamaguti (1975). Para cada uno de los morfotipos de cercarias encontrados en las especies de moluscos, se determinó la prevalencia de infección parasitaria (PIP) (Margolis et al. 1982):
Exposición experimental de alevines a moluscos: En la Estación Experimental acuícola Enrique Jiménez Núñez, en Cañas, Guanacaste se hizo recolecta de moluscos en tres estanques de tierra con dimensiones de $300 \mathrm{~m}^{2}$. Los alevines de tilapia gris utilizados en el experimento (1.5-3.5cm de longitud y $0.8-1.0 \mathrm{~g}$ de peso) fueron reproducidos en piletas de concreto y generados por reproductores sembrados en una proporción de $2: 1$. Antes de cada exposición experimental, se realizó la necropsia a 384 alevines de la cohorte de animales. El tamaño de la muestra se estimó utilizando el software EPIINFO, versión 6,0 (Dean et al. 1995) fijando una prevalencia del $50 \%$, un error absoluto esperado del $5 \%$ y un nivel de confianza del $95 \%$. Se utilizaron acuarios de vidrio de $20 \mathrm{~L}$ de capacidad, provistos de aireadores artificiales. Durante el experimento, los parámetros físicos-químicos de los acuarios se mantuvieron dentro de los rangos según lo recomendado por la Asociación Americana de Tilapia (ATA 1997). La alimentación de los alevines, durante la primera y segunda exposición experimental, se distribuyó en tres raciones (10:00, 14:00 y 17:00 horas), tomando como referencia una tabla de alimentación establecida por ATA.

Diseño de la primera exposición experimental de alevines a moluscos: Cinco alevines de tilapia gris fueron expuestos a cada especie de molusco encontrada en los tres estanques. La proporción molusco-alevín fue de 2:1 (diez moluscos-cinco alevines). Además, se contó con un acuario control (cinco tilapias sanas sin exposición a moluscos). Durante el experimento se mantuvo a los alevines en observación, para documentar la aparición de síntomas clínicos y al morir fueron analizados mediante examen externo y necropsia. Los alevines sanos y los controles se sacrificaron diez

$$
\mathrm{PIP}=\frac{\text { Número hospedadores intermediarios primarios infectados x } 100}{\text { Número hospedadores intermediarios primarios examinados }}
$$


días después de la exposición a los moluscos e igualmente se analizaron mediante examen externo y necropsia. Para los acuarios en los que hubo manifestación de síntomas clínicos o muerte de alevines, se diseñó una segunda exposición experimental.

Diseño de la segunda exposición experimental de alevines a moluscos: Un total de 25 alevines de tilapia gris fueron expuestos a 50 moluscos de una misma especie en cinco acuarios (repeticiones) y un acuario control (5 alevines sin exposición a moluscos). Se observó a los alevines durante el experimento para documentar la aparición de síntomas clínicos $\mathrm{y}$ al morir, fueron analizados mediante examen externo y necropsia. Cinco días post-mortem del último alevín expuesto, se sacrificaron y analizaron a los alevines control.

Examen clínico, anatomopatológico y parasitológico de los alevines: Los alevines expuestos a moluscos estuvieron en observación para documentar y describir los síntomas clínicos. En todos los alevines se analizó la presencia y forma de quistes de metacercarias en tegumento y aletas mediante un examen externo y en branquias mediante necropsia. Dichas estructuras fueron observadas también al estereoscopio y las branquias, adicionalmente al microscopio. En el segundo experimento se calculó la abundancia e intensidad media de metacercarias en branquias (Margolis et al. 1982), extraídas utilizando microagujas y micropinzas. Posteriormente fueron lavadas en solución salina fisiológica $(0.65 \%)$, fijadas en formol caliente al 4\% (Arizmendi 1992); después se realizó la medición y esquematización de las mismas, mediante una cámara clara adaptada a un microscopio fotónico a un aumento de 100X, para ser comparadas con la información contenida en la guía de identificación de tremátodos de Yamaguti (1975). Los moluscos que ocasionaron síntomas clínicos o muerte de los alevines en el segundo experimento fueron analizados mediante las técnicas recomendadas por Stockkopf (1992) y clasificados según Krull (1986) y Yamaguti
(1975), para asociar las metacercarias presentes en los alevines con los morfotipos de cercarias encontrados en moluscos.

\section{RESULTADOS}

Se recolectaron 907 moluscos pertenecientes a cinco especies (Guanacaste: 190, Alajuela: 99, Limón: 142, San José: 181, Heredia: 106, Cartago: 83, Puntarenas: 106). Se identificaron los gastrópodos Melania tuberculata y Melanoides turricula de la familia Thiaridae, Pomacea flagellata (Pilidae) y Haitia cubensis (Physidae) (Fig. 1A-D). El bivalvo encontrado correspondió a Anodontiles luteola (Mycetopodidae) (Fig. 1E). Los moluscos identificados en los diferentes estanques en el territorio nacional se distribuyeron geográficamente tal y como se observa en la Figura 2. En todos los estanques se encontró P. flagellata y M. turricula. En el estanque de la provincia de San José se encontró además $H$. cubensis, mientras que en los de las provincias de Limón, Heredia y Puntarenas se encontró A. luteola además de P. flagellata y M. turricula. Únicamente en el estanque analizado en la provincia de Guanacaste se encontraron las cinco especies de moluscos.

Se determinaron las cercarias presentes en los moluscos estudiados, de tipo distoma, identificándose siete diferentes morfotipos: Xifiodiocercaria, Equinostoma, Oftalmocercaria, Parapleurolofocercus, Cistocerca, Furcocercaria y Leptocercaria. El número y los morfotipos de cercarias encontrados, así como la prevalencia de infección parasitaria de los moluscos, se presentan en los Cuadros 1 y 2. En P. flagelata y M. turricula se lograron determinar un total de cinco morfotipos en total (cistocercaria, equinostoma, xifidiocercaria, oftalmocercaria y leptocercaria), mientras que en H. cubensis se determinaron cuatro morfotipos (equinostoma, leptocercaria, cistocercaria, oftalmocercaria) y tres en A. luteola (cistocercaria, equinostoma y leptocercaria). En $M$. tuberculata se pudo determinar únicamente un morfotipo (parapleurolofocercus).

En la primera exposición de los alevines a las cinco especies de moluscos, solamente 

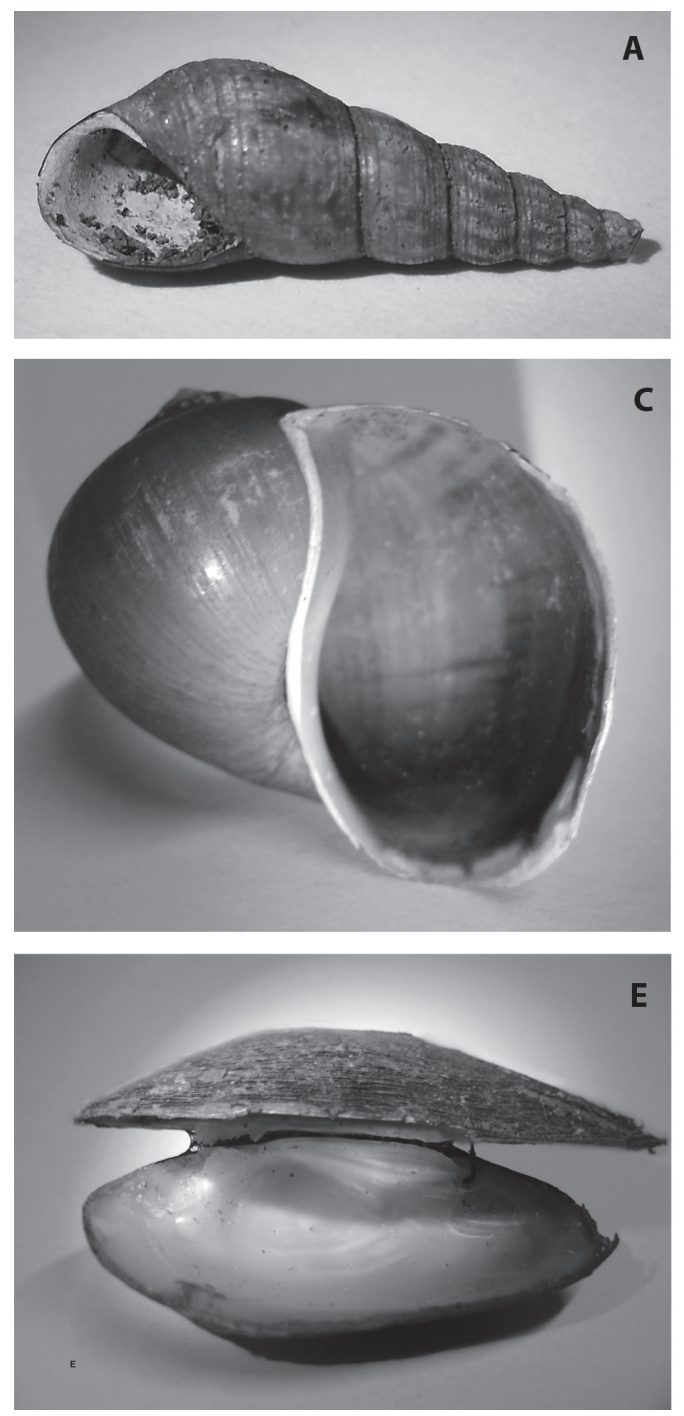

Fig. 1. (A) M. tuberculata, (B) M. turricula. (C) $P$. flagellata. (D) H. cubensis. (E) A. luteola.

los que estuvieron expuestos a $M$. tuberculata presentaron curvamiento corporal, nadado curveado, errático y en espiral; y síntomas clínicos como disnea, letargia, apertura de opérculos y enrojecimiento de branquias 1.5 horas post-exposición. Se observó generalización de boca abierta y muerte universal en un lapso aproximado de 2.5 horas. El examen externo reveló la presencia de pocos quistes en aletas y
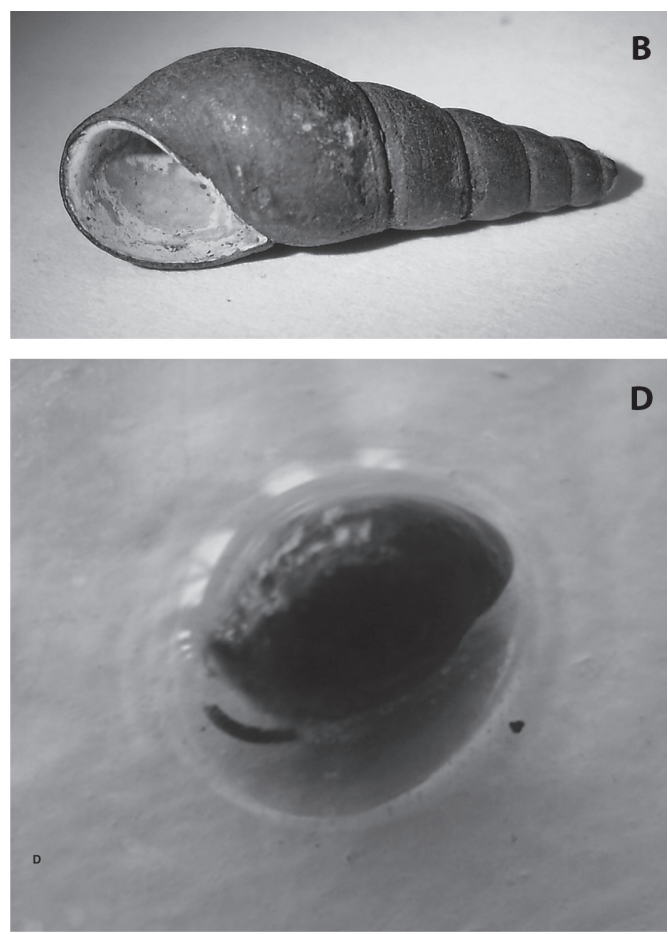

tegumento y la necropsia evidenció gran cantidad de metacercarias en $\operatorname{arcos} y$ filamentos branquiales. Durante los diez días de observación no se determinaron signos clínicos, ni se encontraron quistes en los alevines control ni en los alevines expuestos a otros moluscos.

En la segunda exposición de los alevines a $M$. tuberculata, los peces presentaron los mismos signos clínicos y murieron 2.5 horas p.e. El examen externo reveló la presencia de aproximadamente tres quistes de metacercarias en aletas, dos en tegumento y un promedio de 1026 (abundancia 1018-1027) de digéneos por branquia en cada pez parasitado. En los alevines del acuario control no se encontraron quistes ni se observaron síntomas clínicos durante los cinco días de observación.

La morfometría determinada en 35 metacercarias fue: Cuerpo ovalado a piriforme, de $0.282 \mathrm{~mm}(0.244-0.321)$ de largo por $0.085 \mathrm{~mm}$ (0.083-0.087) de ancho; superficie corporal completamente armada con espinas pequeñas; ventosa oral terminal de $0.037 \mathrm{~mm}(0.026$ $0.056)$ de largo por $0.048 \mathrm{~mm}(0.030-0.051) \mathrm{de}$ 


\section{CUADRO 1}

Especies de moluscos, número de moluscos recolectados y número de morfotipos de cercarias identificados en los moluscos en los estanques de producción de tilapias en las diferentes provincias en Costa Rica

TABLE 1

Freshwater mollusk species, number of freshwater mollusks collected and number of morphotypes of cercariae identified in freshwater mollusks in ponds of tilapia production in different provinces in Costa Rica

\begin{tabular}{lccccccc}
\multicolumn{1}{c}{ Especie } & $\begin{array}{l}\text { Estanque 1 } \\
\text { (Nmr/Nmt) }\end{array}$ & $\begin{array}{c}\text { Estanque 2 } \\
(\mathrm{Nmr} / \mathrm{Nmt})\end{array}$ & $\begin{array}{c}\text { Estanque 3 } \\
(\mathrm{Nmr} / \mathrm{Nmt})\end{array}$ & $\begin{array}{c}\text { Estanque 4 } \\
(\mathrm{Nmr} / \mathrm{Nmt})\end{array}$ & $\begin{array}{c}\text { Estanque 5 } \\
(\mathrm{Nmr} / \mathrm{Nmt})\end{array}$ & $\begin{array}{c}\text { Estanque 6 } \\
(\mathrm{Nmr} / \mathrm{Nmt})\end{array}$ & $\begin{array}{c}\text { Estanque } 7 \\
(\mathrm{Nmr} / \mathrm{Nmt})\end{array}$ \\
P. flagellata & $50 / 3$ & $87 / 2$ & $39 / 3$ & $62 / 3$ & $26 / 1$ & $54 / 4$ & $26 / 1$ \\
M. turricula & $25 / 2$ & $12 / 2$ & $81 / 2$ & $19 / 2$ & $52 / 4$ & $29 / 2$ & $52 / 4$ \\
H. cubensis & $50 / 1$ & $*$ & $*$ & $100 / 4$ & $*$ & $*$ & $*$ \\
A. luteola & $10 / 0$ & $*$ & $22 / 2$ & $*$ & $28 / 3$ & $*$ & $28 / 3$ \\
M. tuberculata & $55 / 1$ & $*$ & $*$ & $*$ & $*$ & $*$ & $*$ \\
TOTAL MOLUSCOS & 190 & 99 & 142 & 181 & 106 & 83 & 106
\end{tabular}

Estanque 1: Guanacaste, Estanque 2: Alajuela, Estanque 3: Limón, Estanque 4: San José, Estanque 5: Heredia, Estanque 6: Cartago, Estanque 7: Puntarenas.

Pond 1: Guanacaste, Pond 2: Alajuela, Pond 3: Lemon, Pond 4 San Jose, Pond 5: Heredia, Pond 6: Cartago, Pond 7: Puntarenas.

*=No se encontró moluscos. Nmr=Número de moluscos recolectados. Nmt=Número de morfotipos identificados.

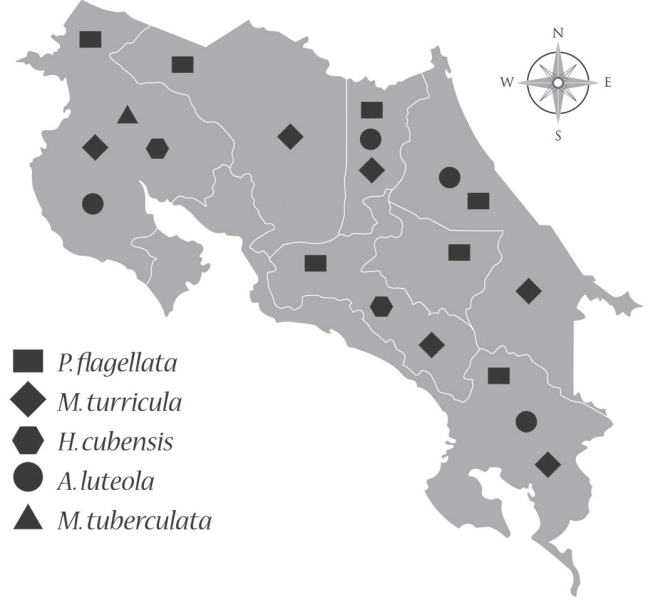

Fig. 2. Distribución de las especies de moluscos identificadas en estanques de producción de tilapia en diferentes provincias de Costa Rica.

Fig. 2. Distribution of freshwater mollusk species identified in tilapia production ponds in different provinces of Costa Rica. ancho; boca situada al centro de la ventosa oral, rodeada por dos coronas provistas de 16 espinas cada una para un total de 32 ; ventosa oral 0.041 mm (0.036-0.059) de diámetro; acetábulo esférico $0.037 \mathrm{~mm}(0.029-0.040)$ de largo por $0.039 \mathrm{~mm}$ (0.034-0.043) de ancho, dispuesto post-ecuatorialmente en la línea media ventral del cuerpo; prefaringe corta, conectada con una faringe musculosa $0.020 \mathrm{~mm}(0.008-0.024) \mathrm{de}$ largo por $0.008 \mathrm{~mm}(0.007-0.010)$ de ancho; esófago corto $0.024 \mathrm{~mm}(0.013-0.034)$, dividido en dos ciegos rectos que terminan a nivel de acetábulo; testículos laterales, localizados en la región posterior del cuerpo; testículos esféricos, el derecho con un diámetro de $0.051 \mathrm{~mm}$ e izquierdo de $0.046 \mathrm{~mm}$; ovario destral, pretesticular; vesícula excretora oscura con forma de "X", con numerosos corpúsculos calcáreos en su interior; poro excretor subterminal. Con base en la morfometría de las metacercarias se determinó que el tremátodo que parasitó a 


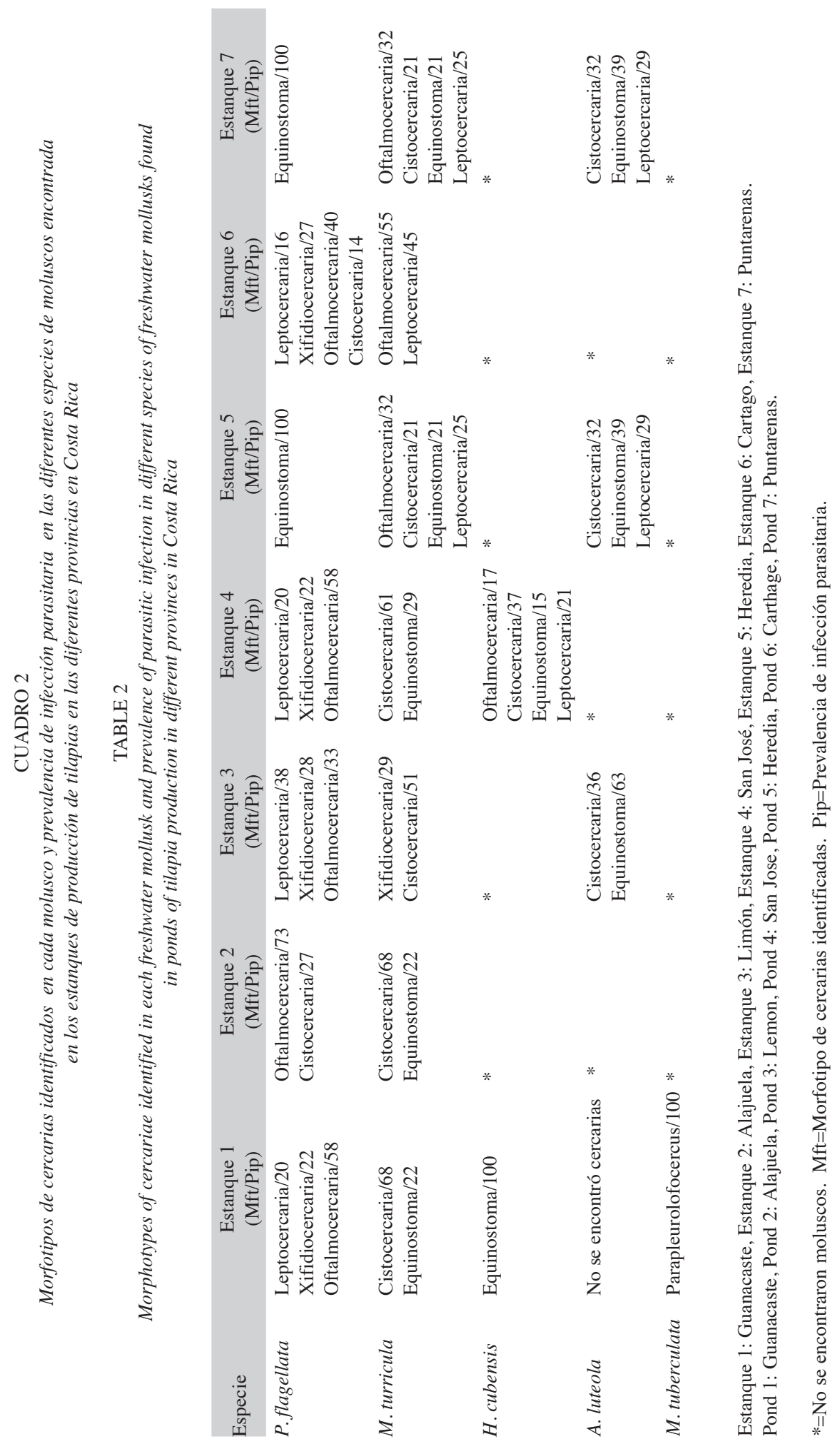


A

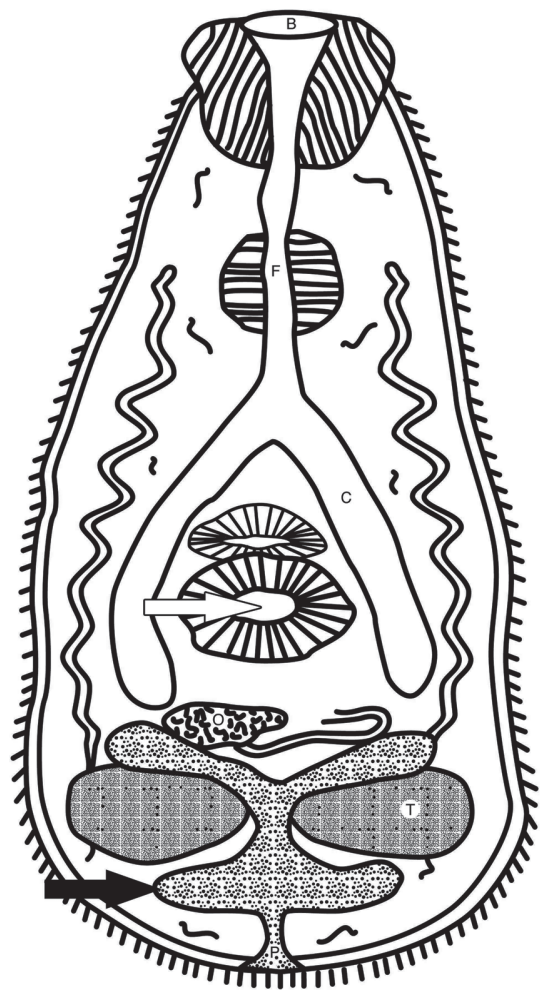

B

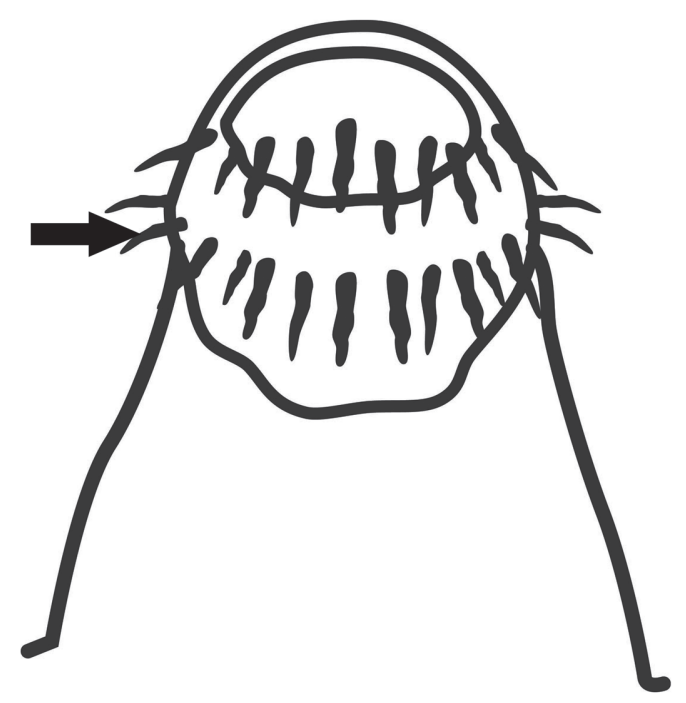

Fig. 3. (A) Metacercaria de C. formosanus liberada del quiste con vesícula excretora en forma de "X" (flecha). Poro excretor subterminal (P). Testículo lateral (T). Oviducto (O). Ciego pilórico (C). Ventosa ventral (flecha blanca). Faringe (F). Ventosa oral terminal (V). Boca subterminal. (B) Ventosa oral cubierta por 32 espinas circunmorales dispuesta en dos filas (flecha).

Fig. 3. (A) Metacercariae de C. formosanus emerged of cyst with excretory vesicle is " $\mathrm{X}$ " shaped (arow). Excretory pore subterminal (P). Lateral testicle lateral (T). Oviduct (O). Caeca (C). Ventral sucker (white arrow). Pharynx (F). Oral sucker terminal (V). Mouth subterminal. (B) Oral sucker covers by 32 circumoral spines in two files (flecha).

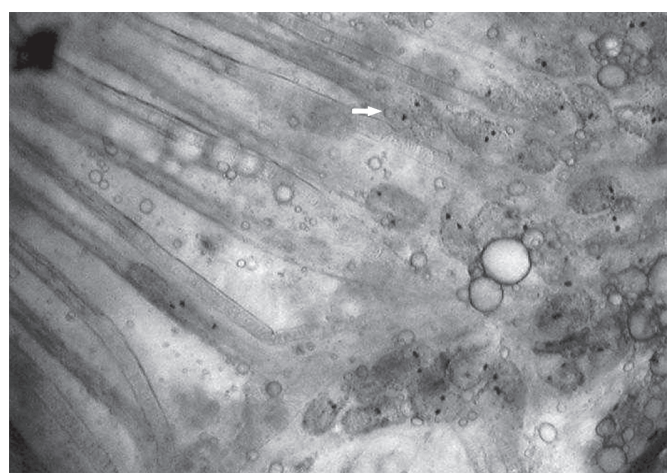

Fig. 4. Filamentos branquiales de un alevín de tilapia gris, experimentalmente infectado con múltiples metacercarias de C.formosanus (flecha).

Fig. 4. Branquial filaments of gray tilapia fry experimentally infected with multiple metacercariae of $C$. formosanus (arrow). 
los alevines fue Centrocestus formosanus (Fig. $3 \mathrm{~A}$ y Fig. 4). La diferencia entre las especies a nivel de metacercarias se realiza con base en el número de espinas orales que presentan los ejemplares, las dimensiones generales del cuerpo y otras estructuras, forma y tamaño de los testículos y ovarios. Los ejemplares descritos en este trabajo corresponden a $C$. formosanus tomando como referencia la descripción de Nishigori (1924). Considerando el número de espinas orales, los ejemplares de metacercarias, presentaron de manera constante 32 espinas distribuidas en una doble corona de 16 espinas, todos los números incluidos en el intervalo establecido por autores como Chen (1942), Kobayasi (1970) y Premvati \& Pande (1974), quienes indicaron la existencia de una doble corona con una variación entre 30 y 36 . Por otro lado presenta una vesícula excretora con forma de " $X$ " que característicamente se ha descrito para esta especie (Pande \& Sukla 1972, Chen 1942, Premvati \& Pande 1974). Continuando con los rasgos taxonómicos, es importante observar el estadio que antecede a la metacercaria. Al observar las cercarias encontramos que del número de espinas orales que comentamos anteriormente, $C$. formosanus difiere de las demás especies descritas para el género Centrocestus, de $C$. armatus, por sus dimensiones corporales, ya que mientras ésta especie alcanza una talla de $0.600 \mathrm{~mm}$ de largo, nuestros ejemplares tienen una talla máxima de $0.282 \mathrm{~mm}$, de $C$. cuspidatus principalmente porque éste presenta una vesícula excretora en forma de "Y", de C. yokogawai por presentar una vesícula excretora en forma de " $\mathrm{H}$ " (rasgo por el que también se distingue de $C$. asadai) y un ovario triangular, característica que a su vez también permite diferenciarlo de $C$. polyspinosus, de acuerdo con Yamagutti (1975). Es relevante destacar la importancia que tiene la descripción de las larvas cercarias y metacercarias, dado que éstas constituyen un estado natural de desarrollo de los trematodos, que pueden proporcionar datos de carácter específico basados en su morfología y fisiología.

En los moluscos M. tuberculata analizados en el segundo experimento se encontraron

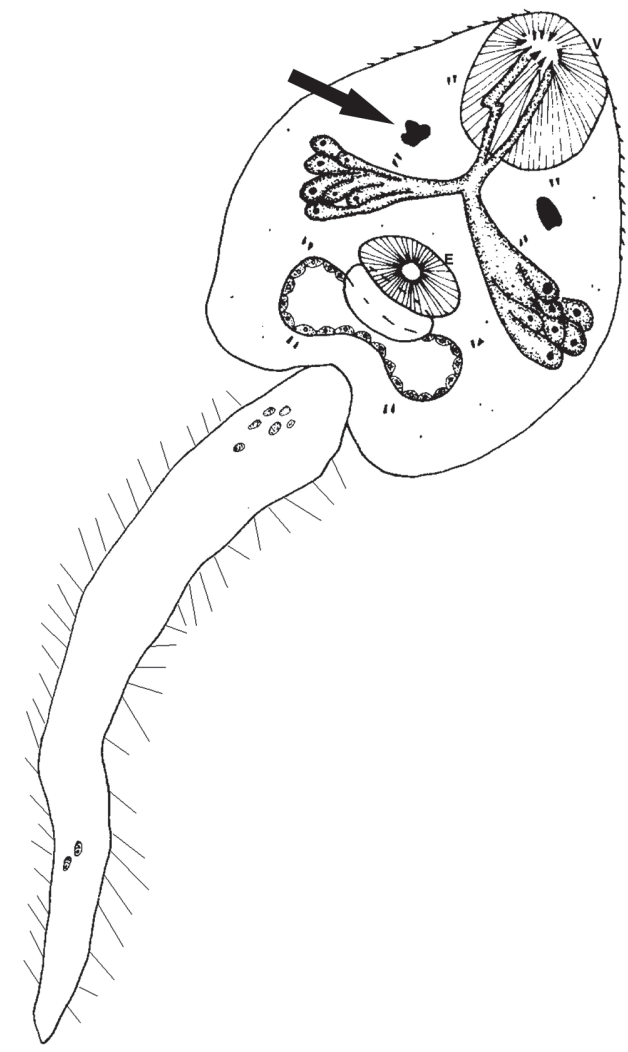

Fig. 5. (A) Cercaria de C. fomosanus, morfotipo parapleurolofocercus tipo distoma, con células en flama (C). Ocelos (flecha). Ventosa oral subterminal (V). Acetábulo rudimentario (E).

Fig. 5. (A) C. fomosanus cercariae, morfotype parapleurolofocercus type distome, with flame cells (C). Ocular spots (arrow). Oral sucker subterminal (V). Acetabulam rudimentary (E).

únicamente cercarias del morfotipo parapleurolofocercus (Fig. 5A).

\section{DISCUSIÓN}

Este es el primer reporte sobre especies de moluscos presentes en estanques de cultivo de tilapias en Costa Rica. En la lista preliminar de moluscos dulceacuícolas del país, publicada por Taylor en 1993, se mencionan P. flagellata, T. tuberculata sinónimo de Melanoides tuberculata (Aguilar et al. 2007) y A. luteola. 
En esta investigación se detectaron dos especies adicionales: $H$. cubensis y $M$. turricula. El primero distribuyéndose ampliamente en el oeste de la India, América Central, Norte y Suramérica y el segundo originario de Filipinas y en conjunto con Melanoides tuberculata consideradas una variación ecológica de una misma especie (Thompson 1984). Estos moluscos se consideraban exóticos para Costa Rica, pero pueden haber sido introducidos al país mediante envíos de peces de la isla de Taiwán (Monge 1997); lo que señala la importancia de examinar los envíos de nuevas líneas genéticas de tilapias al país. La introducción de moluscos exóticos puede ocasionar desplazamiento de las especies nativas o portar helmintos, que podrían afectar a peces autóctonos, animales domésticos y silvestres e inclusive afectar la salud humana.

Aunque el muestreo de moluscos no fue realizado en forma representativa para los estanques de cada una de las provincias, se determinaron diferentes distribuciones de especies de moluscos en las localidades muestreadas. En el estanque ubicado en Guanacaste se encontraron a todas las especies de moluscos. Debido a que dicha finca suple la semilla a las restantes fincas productivas analizadas, se esperaba encontrar todas las especies de moluscos en los estanques de las otras fincas. La distribución variada de especies de moluscos en las diferentes localidades de Costa Rica puede verse influenciada por parámetros físico-químicos del agua y factores ambientales, que limitan la distribución geográfica de los moluscos en el país. Según Cruz \& Flores (1992), factores ambientales tales como temperatura del agua, acidez, transparencia, oxígeno disuelto y luz, influyen de forma importante en la sobrevivencia de los moluscos, determinando así su cobertura y nichos disponibles.

Se presenta además el primer reporte taxonómico de cercarias parasitando moluscos en estanques de cultivos de tilapias en Costa Rica. En total se identificaron siete morfotipos de cercarias parasitando a cinco especies de moluscos, que presentaron, generalmente, parasitismo múltiple. En todos los casos se determinó, sin embargo, dominancia de una especie sobre la otra, lo que se ve reflejado en la prevalencia de infección parasitaria, destacándose infecciones dominantes de Leptocercarias y Xifidiocercarias en las cinco especies de moluscos. Este hallazgo concuerda con el de Olsen (1974), quien observó que en moluscos doblemente infectados por cercarias de Echinostoma trivolvis y Haematoloechus longiplexus, ocurría un dominio del primer tremátodo.

Finalmente se reporta por primera vez la presencia de Centrocestus formosanus en Costa Rica, determinando experimentalmente a Melania tuberculata como hospedero intermediario primario y a la tilapia gris como hospedero intermediario secundario de este tremátodo. Hasta la fecha, solamente una especie de heterófido (Euryhelmis costaricensis) había sido reportada por Brenes et al. 1960 (ver Rodríguez-Ortíz et al. 2004a, 2004b).

Las características morfométricas de las metacercarias observadas en los alevines de Tilapia, coinciden con Nishigori (1924), con las descritas en peces de cultivos en Taiwán y con las de Chen (1942), Yamaguti (1958), Kobayasi (1970), Premvati \& Pande (1974) y Pande \& Sukla (1972). Además coinciden morfológicamente con los quistes del morfotipo 1 encontrados por Muñoz (2001) en branquias e hígado de alevines de tilapias producidos en una finca comercial en Guanacaste. En los moluscos $M$. tuberculata analizados, se encontraron únicamente cercarias del morfotipo parapleurolofocercus, que concuerdan con el hallazgo del parásito $C$. formosanus en los alevines. Dicho hallazgo se dio exclusivamente en los estanques de la finca localizada en Guanacaste.

En la presente investigación, este heterófido produjo una alta mortalidad de alevines, posiblemente por el efecto del área de exposición en los acuarios. Según Hoffman (2000), pocas emigraciones de cercarias de $C$. formosanus producen daños mecánicos y hemorragias en peces de aguas tropicales. Vogelbein \& Overstreet (1988) señalan que el parásito induce una inusual respuesta inflamatoria, 
caracterizada por proliferación de fibroblastos, formando una encapsulación continua alrededor del parásito, que destruye la arquitectura del tejido branquial. Esto coincide con los hallazgos anatomopatológicos actuales.

La Centrocestiasis es una enfermedad originaria de Asia y ha sido reportada en ríos y estanques de cultivos de peces de agua dulce en Estados Unidos, también existen reportes en México y Venezuela, a los que habría sido introducida mediante la importación de caracoles y peces (Arizmendi-Espinosa 1992, Scholz \& Salgado-Maldonado 2000, Hernández et al. 2003, Mitchell et al. 2005). Vogelbein \& Overstreet (1988) estimaron las pérdidas anuales ocasionadas por este parásito en granjas de peces ornamentales juveniles en Estados Unidos en $\$ 4.5$ millones, por consiguiente, el impacto económico que podría estar ocasionando este helminto en la industria acuícola de Tilapia de Costa Rica podría ser muy significativo. Este tremátodo ha sido reportado además en cíclidos, carácidos y ciprínidos silvestres en ríos de Florida, Estados Unidos (Mitchell et al. 2005). Finalmente, $C$. formosanus es considerado zoonótico, como otros helmintos de la familia Heterophyidae y se han reportado casos de infección en humanos en Formosa (Chen 1942), en Japón (Komiaya \& Suzuki 1996) y en Tailandia (Waikagul et al. 1997). Este dato es relevante ya que puede afectar la salud de los costarricenses y causar problemas sanitarios severos, debido a que se acostumbra en Costa Rica consumir la tilapia preparada en jugo de limón o después de secada al sol.

En el presente trabajo se reporta por primera vez en Costa Rica el parásito zoonótico $C$. formosanus y sus hospedadores intermediarios primarios (M. tuberculata) y secundarios (Oreochromis niloticus). Tomando en cuenta que $C$. formosanus se detectó en alevines de tilapia y causó alta mortalidad, futuros estudios son requeridos para identificar su hospedador definitivo con lo que se concluiría el ciclo de vida, evaluar el impacto económico en fincas productoras de tilapias en Costa Rica y generar posibles estrategias de control del parásito. Además, se recomienda controlar la entrada de más moluscos exóticos a Costa Rica y determinar el riesgo de $C$. formosanus en la salud humana.

\section{AGRADECIMIENTOS}

A Luis García Prieto por la visita a Costa Rica y dar sus aportes en la confirmación del diagnóstico de Centrocestus formosanus. A David Osorio Sarabia y Rogelio Rosas Valdez, quienes lo acompañaron en la visita al antiguo CURDTS. A Gabriela Solano Trejos por el apoyo brindado para la conservación de la muestra de Centrocestus formosanus en la Colección Nacional de Helmintos de Costa Rica (CHCR), Facultad de Microbiología, Universidad de Costa Rica, San José.

\section{RESUMEN}

Centrocestus formosanus es un parásito tremátodo zoonótico originario de Asia asociado con muertes de peces principalmente de cultivo. 907 moluscos provenientes de estanques sembrados con tilapias, seleccionados uno por provincia fueron identificados al nivel taxonómico especifico. Se identificaron cuatro gastrópodos y un bivalvo: M. tuberculata, M. turricula, P. flagellata, H. cubensis y A. luteola. Se reporta, por primera vez, la presencia de dos especies de moluscos en Costa Rica. Se identificaron siete morfotipos de cercarias parasitando las cinco especies de moluscos encontradas. En la segunda exposición experimental se demostró que el morfotipo parapleurolofocercus encontrado en M. tuberculata concuerda con el hallazgo de C. formosanus en alevines de tilapia, después del examen clínico, anatomopatológico y parasitológico realizado a los alevines expuestos. Las metacercarias fueron extraídas del quiste utilizando microagujas y micropinzas lavadas en solución salina fisiológica $(0.65 \%)$, fijadas en formol caliente al $4 \%$ y después esquematizadas con una cámara clara adaptada a un microscopio fotónico, estimándose una abundancia e intensidad media de 1018-1027 digeneos por branquia en cada pez parasitado, determinándose así el hospedador intermediario primario y secundario del parásito. En el presente trabajo se reporta por primera vez Centrocestus formosanus en Costa Rica.

Palabras claves: Centrocestus formosanus, Oreochromis niloticus, alevines, moluscos de agua dulce, Costa Rica.

\section{REFERENCIAS}

Aguilar, V., A. Aguirre, J. Alarcón, A. Boomer, S. Contreras \& E. Del Val. 2007. Especies invasoras de alto 
impacto a la Biodiversidad. Nature Conservancy, Jiutepec, Morelos, México.

Arizmendi, E. 1992. Descripción de algunas etapas larvarias de la fase adulta de Centrocestus formosanus de Tezontepec de Aldama, Hidalgo. Zool. 63: 1-11.

ATA (American Association Tilapia). 1997. Tilapia situation and outlook report. Aquaculture Magazine 24: 8-15.

Burch, J.B. \& A. Cruz Reyes. 1987. Clave genérica para la identificación de gastrópodos de agua dulce en México. Instituto de Biología, México, Distrito Federal, México.

Chen, H.T. 1942. The metacercaria and adult of Centrocestus formosanus (Nishigori, 1924), with notes on the natural infection of rats and cats with $\mathrm{C}$. Armatus (Tanabe, 1922). J. Parasitol. 28: 285-298.

Conroy, G. \& D.A. Conroy. 1998. Enfermedades y parásitos de cachamas, pacus y tilapias. Pharma-fish, Maracay, Aragua, Venezuela.

Cremonte, F. \& S.R. Martorelli. 1998. Description of a new species of Maritrema (Digenea: Microphallidae) in Larus dominicanus (Aves: Laridae) from Buenos Aires coast, Argentina. Folia Parasitological. 45: 230-232.

Cruz, F.M. \& F.A. Flores. 1992. Mollusks'distribution and environmental characterization in the river plumes of the gulf of México. Secretaria de Pesca, Cancum, Quintana Roo, México.

Dean, A.G., J.A. Dean, D. Coulombier, K.A. Bredel, D.C. Smith, A.H. Burtom, R.C. Dicker, K. Sullivan, R. Fagan \& T.G. Arner. 1995. Epi Info, Versión 6: A Word Processing, Database, and Statistics Program for Public Health on IBM-compatible Microcomputers. Center for Disease Control and Preventions, Atlanta, Georgia, EEUU.

FAO. 2005. El estado mundial de la pesca y la acuicultura. FAO, Roma, Italia.

Healy, G. 1985. Trematodes transmittted to man by fish, frogs and crustacea. Wild. Dis. 6: 255-261.

Hepher, B. \& Y. Pruginin. 2004. Cultivo de tilapia en estanques bajo condiciones controladas. ICLARM, Manila, Filipinas.

Hernández, L.E., M. Díaz \& A.K. Bashirllah. 2003. Description of different developmental stages of Cen- trocestus formosanus (Nishigori, 1924) (Digenea: Heterophyidae). Rev. Cient. 13: 285-292.

Hoffman, G.L. 2000. Parasites of North American fresh water fishes. Comstock, Nueva York, Nueva York, EEUU.

Incopesca. 2005. Informe de labores 2004. INCOPESCA, San José, Costa Rica.

Incopesca. 2006. Informe de labores 2005. INCOPESCA, San José, Costa Rica.

Kobayasi, H. 1970. Proposition to find more reasonable classification and unifiable numenclature and the flukes belonging to the subfamily Centrocestinae Looss 1989. J. Parasitol. 4: 19-20.

Knoff, M., S.C. Clemente, R.M. Pinto \& D.C. Gomes. 2001. Digenea and acanthocephala of elasmobranch fishes from the southern coast of Brazil. Aquaculture International 11: 212-221.

Komiaya, Y. \& N. Suzuki. 1996. The metacercariae of trematodes belonging to the family Heterophyidae from Japan and adjacent countries. J. Parasitol. 15: 209-214.

Krull, W.H. 1986. The life history of two North American frog lung flukes. J. Parasitol. 16: 207-212.

Margolis, L., G. Esch, J. Holmes, A. Kuris \& G. Schard. 1982. The use of ecological terms in parasitology. J. Parasitol. 68: 131-133.

Matamoros, R.A. 1982. Observaciones anatomo-patológicas en la Tilapia. Universidad Nacional, Heredia, Costa Rica.

Mitchell, A.J., A.E. Goodwin, M.J. Salmon \& T.M. Brandt. 2005. Spread of an exotic fish-gill trematode: A far reaching and complex problem. Reviews in Fish Health 30: 11-16.

Monge, N.J. 1997. Moluscos de importancia agrícola y sanitaria en el trópico: la experiencia costarricense. Universidad de Costa Rica. San José, Costa Rica.

Muñoz, J.M. 2001. Identificación y prevalencia de parásitos en las primeras etapas de producción en tilapia nilótica Oreochromis niloticus (Pisces: Cichlidae) cultivada intensivamente, en Cañas, Guanacaste. PCVET, Heredia, Costa Rica.

Nishigori, M. 1924. The life cycles of two new species of Heterophyidae, Monorchotrema Taihokui and M. 
taichui found in Formosa. Preliminary note. Taiwan, Igakkai Zasshi. 237: 567-570.

Noga, E.J. 2000. Fish Disease: Diagnosis and Treatment. Acribia, Ames, Iowa, EEUU.

Olsen, O.W. 1974. Animal Parasites: their life cycles and ecology. University Park Press, Baltimore, Maryland, EEUU.

Pande, B. y R. Sukla. 1972. Metacercarial Cyst of Aplorchis pumilio its development in experimental mammals and two other heterophyid infections in fresh water fishes and theirs zoonotic significance. Indian J. Anim. Sc. 42: 971-978.

Paperna, I. 1996. Parasites, infections of fishes in Africa. FAO, Roma, Italia.

Paperna, I. 1991. Diseases caused by parasites in the aquaculture of warn water fish. Ann. Rev. Fish. Dis. 1: $155-194$

Pironet, F.N., J. Jones. 2000. Treatments for ectoparasites and diseases in captive Western Australian fish. Aquaculture International 8: 349-361.

Post, G. 1983. Textbook of Fish Health. TFH, Hong Kong, República Popular China.

Premvati, G \& V. Pande. 1974. On C. formosanus (Nishigori 1924) Price 1932 and its experimental infection in white leghorn Chicks. J. Parasitol. 23: 79-84.

Rodríguez, A. 1980. Moluscos y crustáceos decapados de Venezuela. Ins. Venezolano de Inv. Científicas, Caracas, Venezuela.

Rodríguez O., B., L. García-Prieto \& G. Pérez-Ponce de León. 2004a. Checklist of the helminths parasites of vertebrates in Costa Rica. Rev. Biol. Trop. 52: 313-354.
Rodríguez, O.B., L. García-Prieto, J. Herrera-Vázquez \& G. Pérez-Ponce de León. 2004b. Addendum to the checklist of the helminths parasites of vertebrates in Costa Rica. Rev. Biol. Trop. 52: 355-369.

Scholz, T., \& G. Salgado-Maldonado. 2000. The introduction and dispersal of Centrocestus formosanus (Nishigori, 1924) (Digenea: Heterophyidae) in Mexico. Am. Midl. Nat. 14: 185-200.

Soulsby, E.J.L. 1987. Parasitología y Enfermedades Parasitarias en los Animales Domésticos. Nueva Interamericana, México.

Schmidt, G.D. \& L.S. Roberts. 2000. Trematoda: Form, Functions, and classifications of Digeneans. McGrawHill, Boston, Massachusetts, EEUU.

Taylor, D.W. 1993. Moluscos dulceacuícolas de Costa Rica: Introducción y lista preliminar. Rev. Biol. Trop. 41: 653-665.

Thompson, F. 1984. The Freshwater Snails of Florida, A Manual for Identification. Gainesville, Florida, EEUU.

Volgelbein, D., \& R.M. Overstreet. 1988. Life-history and pathology of a heterophyid trematode infecting Florida-reared ornamental fishes. Fisheries 30: 11-16.

Waikagul, J., T. Wongsaroj, P. Radomyos, V. Meesomboom, R. Praewanich \& D. Jongsuntikul. 1997. Human infection of Centrocestus formosanus in Thailand. Southeast Asian. J. Trop. Med. Public Health 28: 831-5.

Yamaguti, S. 1958. Systema helminthum. Vol. I. The digenetic trematodes of vertebrates. Parts I and II. Interscience, Nueva York, Nueva York, EEUU.

Yamaguti, S. 1975. A synoptical review of life histories of digenetic trematodes of vertebrates with special reference to the morphology of their larval forms. Keigaku, Tokyo, Japón. 